\title{
Analisis fisiográfico para el levantamiento pedológico semidetallado
}

\author{
Osvaldo A. Barbosa ${ }^{1}$, Luis A. Bertani ${ }^{2}$, Fidelia R.H. Fernandez ${ }^{3} \&$ Raúl P. Mendoza ${ }^{4}$
}

1 Area de Recursos Naturales, Facultad de Ingeniería de la Universidad Nacional de San Luis (UNSL), 25 de mayo 384 (5730) V. Mercedes (SL), Argentina, E-mail: barbosa@fices.unsl.edu.ar (Foto)

2 Lantel, Laboratorio Norpatagónico de Teleobservación, UN del Comahue, Argentina.

${ }^{3}$ Ministerio del Ambiente y de los Recursos Naturales Renovables, Trujillo, Venezuela.

4 Plan Nacional de Rehabilitación de Tierras, Colombia.

Protocolo $176-26 / 11 / 2002$ - Aprovado em 7/7/2004

Resumen: Dentro de un levantamiento edafológico semidetallado, se realizó un análisis fisiográfico del área entre los municipios de La Unión, La Victoria, Toro y Obando (Departamento Valle del Cauca, Colombia). El objetivo del trabajo fue la evaluación de la utilización de la metodología del análisis fisiográfico y su clasificación por el sistema CIAF. En el sector se encuentran dos provincias fisiográficas: la Depresión Cauca-Patía y la Cordillera Occidental. En la primera dominan los procesos agradacionales, mientras en la segunda predominan los degradacionales. En la zona plana, los elementos que permitieron el reconocimiento de las unidades de paisaje son: canales de drenaje, uso de la tierra, condición de drenaje y procesos pedogenéticos, que en algunas unidades se manifestaron a partir de cambios de tonalidades observadas por fotointerpretación. En estos sectores el relieve tuvo muy poca importancia debido a que los desniveles en el terreno son de baja significación. En el sector de mayor relieve los elementos que permitieron diferenciar las distintas unidades de paisaje fueron: la configuración de la superficie (relieve y pendientes compuestas), vegetación natural, rocas, patrón de drenaje, material parental y denudación. Se concluye que la metodología aplicada y la clasificación fisiográfica adoptada contribuyeron a entender los procesos que originaron estos paisajes.

Palabras-clave: fotointerpretación, análisis fisiográfico, levantamiento edafológico semidetallado, sistema CIAF

\section{Physiographic analysis as a part of a semidetailed pedologic survey}

\begin{abstract}
As part of a semidetailed pedologic survey, the physiographic analysis of the area comprising the La Unión, La Victoria, Toro and Obando municipalities (Valle del Cauca Department, Colombia) was conducted. The aim of this work was the evaluation of the use of the physiographic analysis and its classification according to the CIAF System. The area includes two physiographic provinces: the Depresión Cauca-Patía (Cauca-Patía Graben) and the Cordillera Occidental (Western Range). Aggradation processes predominate in the former while degradation ones prevail in the latter. In the plains, the elements which enabled the reconnaissance of the landscape units were as follows: drainage channels, land use, drainage conditions and pedogenic processes, which, in some units, manifested through colour changes observed by means of photointerpretation. In these areas, the relief was of minor importance because terrain variations are of little significance. In the mountain region, the elements which enabled the differentiation of the landscape units were as follows: landscape (relief and compound slopes), natural vegetation, rocks, drainage pattern, parent material and denudation. It is concluded that the applied method and the adopted physiographic classification were instrumental in the understanding of the processes originating these landscapes.
\end{abstract}

Key words: photointerpretation, physiographic analysis, semidetailed pedologic survey, CIAF system 


\section{INTRODUCCION}

El relevamiento de suelos es un exámen sistemático en el campo y en el laboratorio, el cual incluye la descripción, clasificación y el mapeo de las clases de suelos, la interpretación para usos múltiples y la predicción de su comportamiento bajo diferentes usos y/o sistemas de manejo (Soil Survey Division Staff, 1993). Comprende varias etapas a saber: a) mapeo, b) descripción y caracterización, c) clasificación y d) interpretación del reconocimiento realizado (Forero et al., 1988; Elbersen et al., 1989; Romero, 1992; Barbosa et al., 2000a y 2000b). La fotografía aérea desempeña un papel fundamental en la primera de estas fases, en donde el uso sistemático de la fotointerpretación es indispensable para la obtención de un mejor resultado, cuali y cuantitativo (Bennema y Gelens, 1976); teniendo en cuenta que la fotointerpretación es el procedimiento para inferir las "relaciones" a partir del análisis visual de las fotos aéreas.

En la génesis de los diferentes tipos de suelo intervienen dos procesos fundamentales, los cuales tienen distinta importancia entre sí según el suelo del que se trate. Ellos son: a) geogénesis: que nos indica el tipo, origen y evolución de los paisajes y sus materiales a partir de los cuales se origina el suelo y b) pedogénesis: que hace referencia a los distintos factores y procesos que han actuado sobre el material parental y que dieron como resultado un suelo determinado (Barbosa et al. 1998).

Por otro lado, el análisis fisiográfico permite entender los procesos que han originado los distintos paisajes de una región, dentro de los cuales habrá cierta homogeneidad en su geogénesis (Barbosa et al., 1997), permitiendo a su vez conocer las características internas de los suelos. De allí se desprende un principio básico del análisis fisiográfico: existe una relación directa entre las propiedades externas de un paisaje y sus características internas. De acuerdo a este principio el Soil Survey Manual (Soil Survey Division Staff, 1993) sostiene que los suelos son perfiles tanto como paisajes.

En consecuencia es posible predecir los distintos perfiles de suelos que se encontrarán en un lugar determinado si se logra entender la fisiografía de esos paisajes. En ello juega un papel fundamental la comprensión de los procesos que actúan sobre los diferentes paisajes y que están condicionados por los factores: clima, tiempo, material parental y relieve. Estos, junto a los organismos del suelo (biota), son los que determinan el origen y evolución de los distintos tipos de suelos. Esta similitud entre los factores formadores de los suelos y de los paisajes refuerza el principio en que se basa el Soil Survey Manual (Soil Survey Division Staff, 1993), de tal forma que para llevar a cabo un buen estudio pedológico será necesario un conocimiento de la fisiografía del lugar. Debido a que el paisaje se nos presenta como un complejo de elementos muy diferentes que pueden alcanzar una diversidad muy grande, si relacionamos las características más sobresalientes de los mismos, reconoceremos a cada uno de ellos (Gonzalez Bernaldez, 1981).

La clasificación fisiográfica del Centro Interamericano de Aerofotointerpretación (CIAF), utiliza una serie de unidades territoriales que se encuentran organizadas jerárquicamente en una disposición piramidal en cuyos vértices están las estructuras geológicas propias de todo continente. La primera categoría del sistema, la provincia fisiográfica, corresponde aproximadamente a la región natural, en la que pueden prevalecer una o más unidades climáticas. Estas están constituídas por conjuntos de unidades genéticas de relieve con relaciones de parentesco de tipo geológico, topográfico y espacial. La segunda, la unidad climática, es una categoría fisiográfica superior que comprende tierras cuyas temperaturas promedio anual y la humedad disponible son lo suficientemente homogéneas como para reflejarse en la génesis específica de los suelos o, al menos, en la cobertura vegetal y en el uso de la tierra. La siguiente, gran paisaje o unidad genética de relieve, abarca asociaciones y complejos de paisajes con relaciones de parentesco de tipo espacial, genético y topográfico definidos, en donde se presenta un alto grado de homogeneidad climática (Villota, 1997).

Los paisajes fisiográficos se definen por: el clima, el material originario y la edad (Botero, 1984), y se caracterizan por los aspectos externos (relieve) e internos (perfiles), típicos para cada paisaje. Villota (1997) los define como porciones tridimensionales de la superficie terrestre resultantes de una misma geogénesis, que pueden describirse en términos de semejantes características climáticas, morfológicas, de material parental y edad, dentro de las cuales puede esperarse una alta homogeneidad pedológica y una cobertura vegetal o un uso de la tierra similar. Ellos se establecen dentro de un Gran Paisaje, en base a su morfología específica, a la cual se le adiciona como atributos: el(los) material(es) parental(es) y/o edad, esta última en términos relativos (muy antiguo, antiguo, subreciente, reciente, subactual, actual) o de niveles (alto, medio, bajo). Es la unidad fundamental, ya que sirve para definir las clases de suelos que tienen características comunes al nivel de generalización taxonómica utilizada en este estudio.

El subpaisaje, unidad que sigue en jerarquía, es una división del paisaje fisiográfico que se realiza con criterios prácticos relacionados con algún cambio en las condiciones de los suelos, y por ende en la cobertura vegetal y uso de la tierra. Generalmente se establecen con base en la posición específica que tienen dentro del paisaje (por ejemplo: cima, hombro, ladera, etc.) y que se califica por el grado y forma de la pendiente, clase y grado de erosión, condición de drenaje, etc (Villota, 1991 y 1992).

El objetivo de este estudio fue analizar y clasificar fisiográficamente (por el sistema CIAF) el área comprendida entre los municipios de La Unión, La Victoria, Toro y Obando (Departamento Valle del Cauca, Colombia), de manera de conocer y explicar la distribución de los cuerpos de suelos dentro del levantamiento edafológico semidetallado de la zona citada.

\section{MATERIAL Y MÉTODOS}

Se utilizaron diferentes materiales de gabinete y de campo. Entre los primeros tenemos: estereoscopio de espejos, fotografías aéreas (vuelos C-2141, C-2415 y C-2418 con escalas aproximadas de $1: 23.000$ a $1: 25.000$ y $1: 43.000$ a 1:45.000), fotografías aéreas ampliadas, cartas topográficas a escala 1:25.000, mapa político del Departamento Valle del Cauca y mapas geológicos de Ingeominas del área de estudio. Los 
equipos de campo son los comunes a todo relevamiento edafológico: barrenos, palas, tabla de color de suelo "Munsell”, etc. (Forero et al., 1988; Barbosa et al., 2000b)

El trabajo fue realizado en diferentes etapas de acuerdo a Barbosa et al. (1998). En la fase preparatoria se evaluó el material bibliográfico disponible relacionado con la geología, geomorfología, climatología, vegetación, agricultura y otros aspectos de interés general de la zona de estudio y se analizaron los mapas e informes de levantamientos de suelos existentes.

En una segunda parte, se realizó el montaje de las fotografías aéreas con el fin de comprobar la zona de cubrimiento y determinar el índice de vuelo del área. Esto permitió obtener una idea preliminar de los grandes paisajes en que se divide la superficie de estudio. Posteriormente se trazaron las líneas de vuelo y de coincidencia y se identificaron los poblados, vías principales, etc. Luego se efectuó la fotointerpretación permitiendo identificar los elementos básicos: pendiente y relieve, vegetación natural, cultivos, características de la superficie del suelo, rocas, etc., los elementos compuestos: patrón de drenaje, uso de la tierra, parcelaje, etc. y los elementos inferidos: condición de drenaje, clima, material parental, etc. A partir de la detección y la delineación de algunos de estos elementos, se hizo posible la confección de un mapa fisiográfico preliminar en donde se reconocieron distintas unidades de terreno con características homogéneas y en donde se utilizó el sistema de clasificación fisiográfica del CIAF hasta el nivel de subpaisaje. Se seleccionaron áreas de muestreo teniendo en cuenta la presencia de los diferentes paisajes, cubriendo aproximadamente entre el 10 y el 15\% de la totalidad del área de trabajo (Figura 1).

Se llevó a cabo un reconocimiento preliminar de la zona, con lo cual se obtuvo una caracterización de los paisajes y de
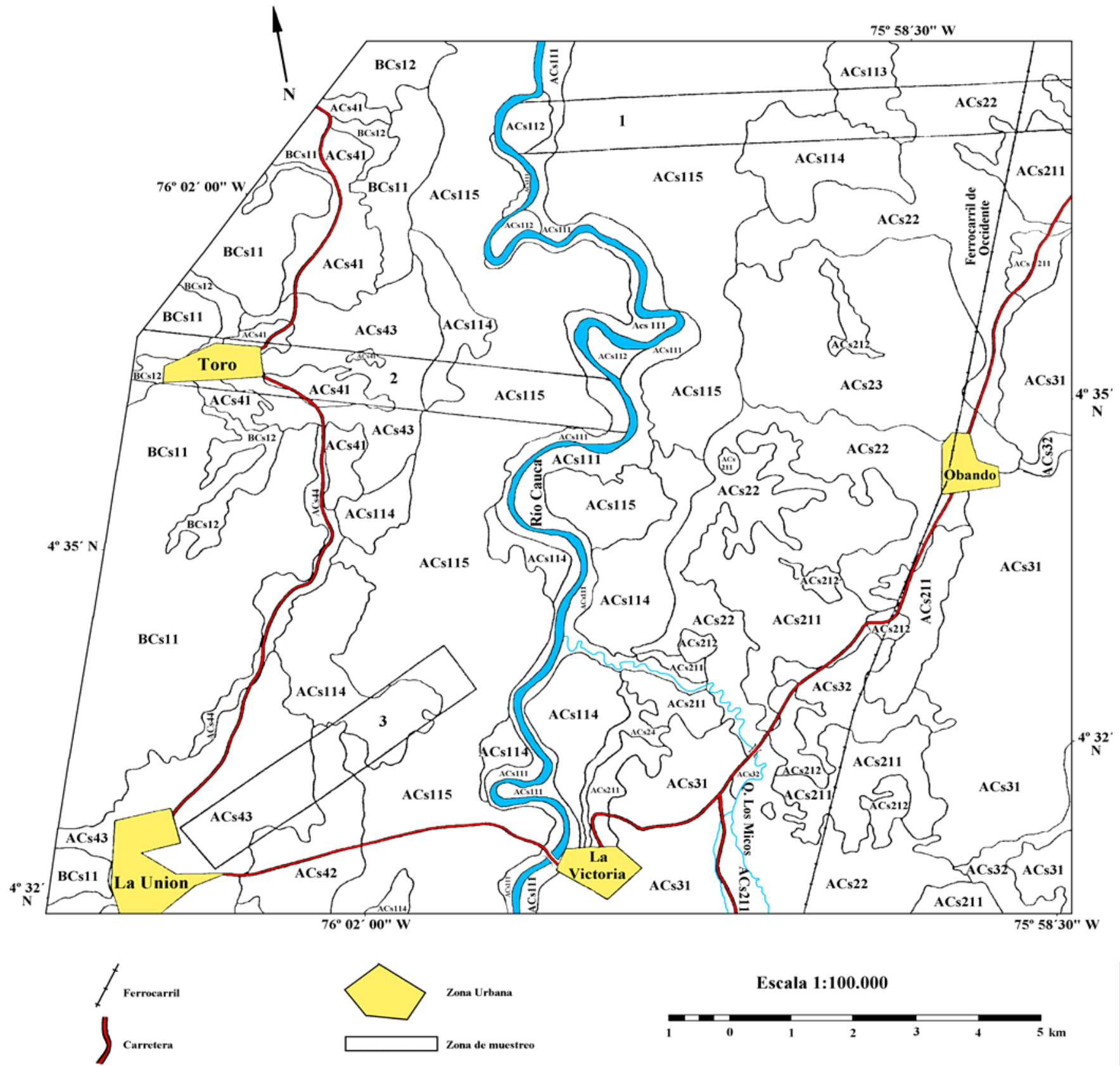

Figura 1. Mapa fisiográfico-edafológico obtenido 
los suelos verficándose la confiabilidad de la fotointerpretación y la validez de la ubicación de las áreas de muestreo.

Posteriormente se realizaron observaciones de tipo detallado en las áreas seleccionadas utilizando el procedimiento standard (Soil Survey Division Staff, 1993). A cada una de ellas se la clasificó de manera tentativa en el campo, de acuerdo a la taxonomía del Soil Survey Staff (1999). Al final del levantamiento se realizó una generalización de los suelos encontrados, combinando las diferentes unidades de mapeo utilizadas: Consociaciones, Asociaciones y Complejos. Las Consociaciones están constituidas por al menos un 75\% de un conjunto de suelos. Las Asociaciones son unidades politáxicas en la cual dos o más conjuntos de suelos se encuentran asociados geográficamente y son lo suficientemente extensas para mapearse por separado en una escala mayor. Los Complejos son también unidades politáxicas pero ocurren en un patrón geográfico tan intrincado que no pueden mapearse individualmente en levantamientos detallados, aún cuando tienen posiciones definidas dentro del paisaje. También se utilizaron los conjuntos de suelos definidos como unidades funcionales abstractas utilizadas a modo de fase, según el paisaje fisiográfico donde ocurra, con nombres comunes a la región.

Luego se realizaron observaciones en levantamiento libre según necesidad, en número suficiente para realizar las comprobaciones de los suelos reconocidos en las áreas de muestreo, o como para caracterizar los suelos definidos en las mismas.

Se procedió a la localización de los perfiles típicos, en donde se realizó un exhaustivo muestreo de los diferentes horizontes de cada uno de ellos para análisis en laboratorio (Soil Survey Laboratory Staff, 1996).

La última etapa consistió en compilar los mapas, clasificar en forma definitiva los suelos encontrados de acuerdo con los resultados de laboratorio y confeccionar el informe respectivo. Se obtuvo un mapa de escala 1:50.000 para el área de trabajo.

Se realizó la determinación del índice de calidad de Legros (Ministerio de Ambiente de España, 2000) que permite realizar un control de la densidad de observaciones y del número de análisis necesarios para una correcta cartografía de suelos, evaluando de esta manera la calidad del mapa de suelo obtenido. Este índice I se basa en los perfiles analizados (Pa), en las observaciones (Pna), en la escala final del mapa $(E)$, en la superficie prospectada en hectáreas (S) y en las unidades cartográficas (U) definidas (Ecuación 1).

$$
I=\frac{2 P a+P n a}{75 E \sqrt{S U}}
$$

\section{Descripción del área de estudio}

La zona de estudio corresponde a la parte plana del valle del río Cauca y algunos sectores montañosos de las Cordilleras Central y Occidental próximas a la misma, en jurisdicción de los municipios de La Unión, La Victoria, Toro y Obando. Se encuentra localizada entre los $4^{\circ} 30^{\prime} 30^{\prime \prime}$ y $4^{\circ} 38^{\prime} 46^{\prime \prime}$ de latitud Norte y $75^{\circ} 57^{\prime} 43^{\prime \prime}$ y $76^{\circ} 03^{\prime} 35^{\prime \prime}$ de longitud Oeste, con un promedio de altura de $920 \mathrm{msnm}$ para el mayor sector de trabajo y con altitudes máximas y mínimas comprendidas entre los 1400 y 900 msnm, respectivamente.

Los registros pluviométricos no superan los $1500 \mathrm{~mm}$ de promedio anual y su distribución es de forma bimodal, siendo los meses más lluviosos marzo, abril y mayo, y septiembre, octubre y noviembre en un segundo período de precipitaciones. Los promedios anuales de temperatura se ubican entre los 23 a $24^{\circ} \mathrm{C}$, no existiendo diferencias significativas entre las máximas y mínimas. Los balances hídricos en forma general muestran un déficit para los meses de enero, febrero y agosto. De acuerdo a las escasas variaciones altitudinales y del gradiente humedad observado en la zona se ha clasificado a la misma como de clima cálido seco (van Wambeke, 1981; García, 1983; Lopez y Olaya, 1985).

El área abarca unas 20.000 ha aproximadamente en donde quedan incluídas dos provincias fisiográficas: la depresión Cauca-Patía y la Cordillera Occidental.

En el Tabla 1 se observa la leyenda fisiográfica-pedológica obtenida correspondiente al mapa del levantamiento (Figura 1).

El valle del río Cauca es una depresión tectónica que separa las Cordilleras Central y Occidental. Su forma actual es el resultado de una serie de levantamientos y hundimientos ocurridos durante el Terciario, conjuntamente con la sedimentación posterior del Cuaternario. La actividad orogénica terciaria ha dado lugar a una serie de anticlinales y sinclinales, algunos de los cuales se encuentran presentes en la región (sinclinal Miravalles y anticlinal próximo a la localidad de Holguín). Ambas estructuras han afectado a rocas de la Formación La Paila (Tobas dacíticas retrabajadas, bancos y lentes arenosos, conglomerados).

Durante el mismo período, el valle del Cauca estuvo ocupado por un lago que en sus inicios tuvo influencias marinas (García, 1983). Posteriormente, el río Cauca, sufrió varios represamientos que según Reese y Goosen (Gomez, 1977) estarían originados en los sucesivos depósitos de los volcanes Nevado del Ruiz, Nevado Santa Isabel y Nevado del Quindío. Esto explica el origen de los sedimentos finos de tipo lacustre que cubren en un 10-15\% el piso del valle y que constituyen el material parental de los suelos de un sector del área (Formación Zarzal). Ellos fueron depositados en forma de grandes abanicos (unidades cartográficas ACs211, ACs212 y ACs41 en Figura 1) que en la actualidad están muy disectados como consecuencia de la acción de agentes exógenos que han actuado durante todo el Pleistoceno. Como resultado de esta acción, en la actualidad las geoformas aparecen configurando lomeríos de cimas redondeadas y laderas cortas, con pendientes que en la mayoría de los casos no superan el 25\%. En general la altura oscila entre 50 y 60 m en relación al piso del valle, registrandosé las mayores en las proximidades de ambas Cordilleras. En algunos sectores estas superficies alomadas han sido cubiertas por sedimentos más jóvenes (cuaternarios) de abanicos aluviales o de la llanura aluvial de desborde del río Cauca, que forma el otro tipo de material parental de los suelos en el área de estudio. 
Tabla 1. Leyenda fisiográfica-pedológica del área de estudio

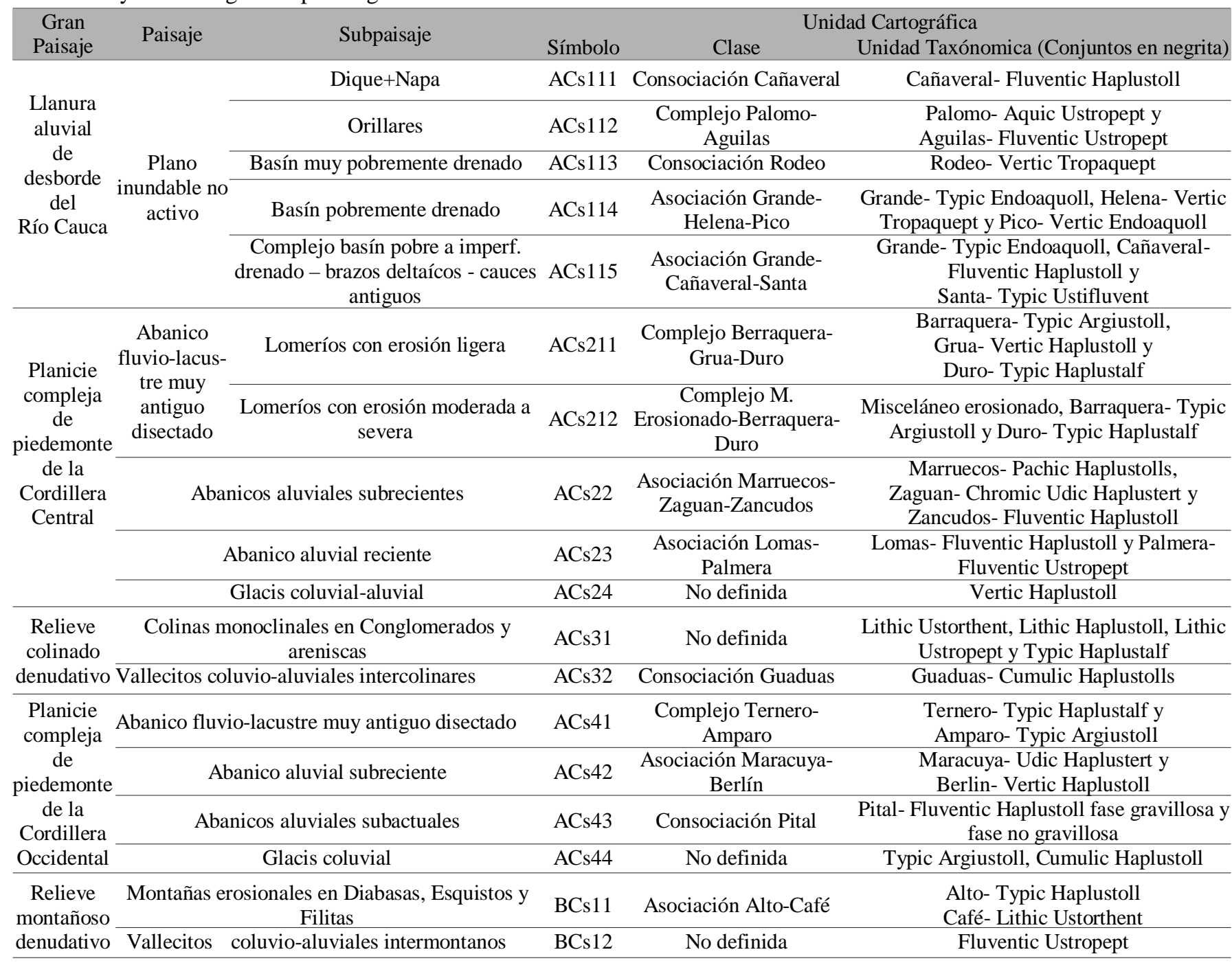

\section{RESULTADOS Y DISCUSIÓN}

La posibilidad de contar con fotografías de la totalidad del área de trabajo, permitió ahorrar tiempo y esfuerzo al hacer posible un buen sentido de la localización y un rápido acceso a los lugares escogidos para las observaciones, utilizando caminos que en muchos casos no figuran en la cartografía de detalle.

El uso de ampliaciones de algunas de las fotografías, posibilitó un mejor nivel de detalle, especialmente en aquellos sectores donde se realizaron las áreas piloto del levantamiento edafológico propiamente dicho. Ello contribuyó a un mejor conocimiento de la relación suelo-paisaje a partir de una mejor visualización de las distintas geoformas y sus variaciones espaciales.

Entre las limitaciones cabe mencionar el hecho de que se contó para este estudio con cuatro fajas de fotografías que presentaban distintas escalas entre sí.

En la zona plana, la fotointerpretación se llevó a cabo teniendo en cuenta los cambios de tono, textura, formas y uso de la tierra, hecho que se puede observar en el mosaico del sector de estudio (Figura 2). En este caso el relieve careció de importancia por tratarse de un área de topografía plana. Debido a esto las líneas que delimitaron las unidades fisiográficas se trazaron en algunos casos con cierta dificultad, a lo que se debe agregar la alta intervención del hombre en estos sectores (por ejemplo: nivelación del terreno para riego, correción del cauce del río, canales de riego, etc.). Algunos de estos límites fueron corregidos siguiendo evidencias netamente pedológicas durante el trabajo de campo.

El desnivel existente entre el plano de sedimentación aluvial del río Cauca y los sectores colinados-montañosos correspondientes a ambas Cordilleras, ha permitido el desarrollo de llanuras de piedemonte conformadas por abanicos aluviales y glacís coluviales de distintas edades. En el piedemonte de la Cordillera Central, se han reconocido abanicos de edad subreciente (unidad cartográfica ACs22 en Figura 1). Estos poseen gran extensión y están constituídos por materiales de tamaños medios en el ápice y cuerpo, y finos y muy finos hacia la zona distal. Esta unidad se reconoce con relativa facilidad en las fotografías aéreas por su posición y por presentar tonos oscuros. En el campo dicha geoforma se reconoció por presentar 


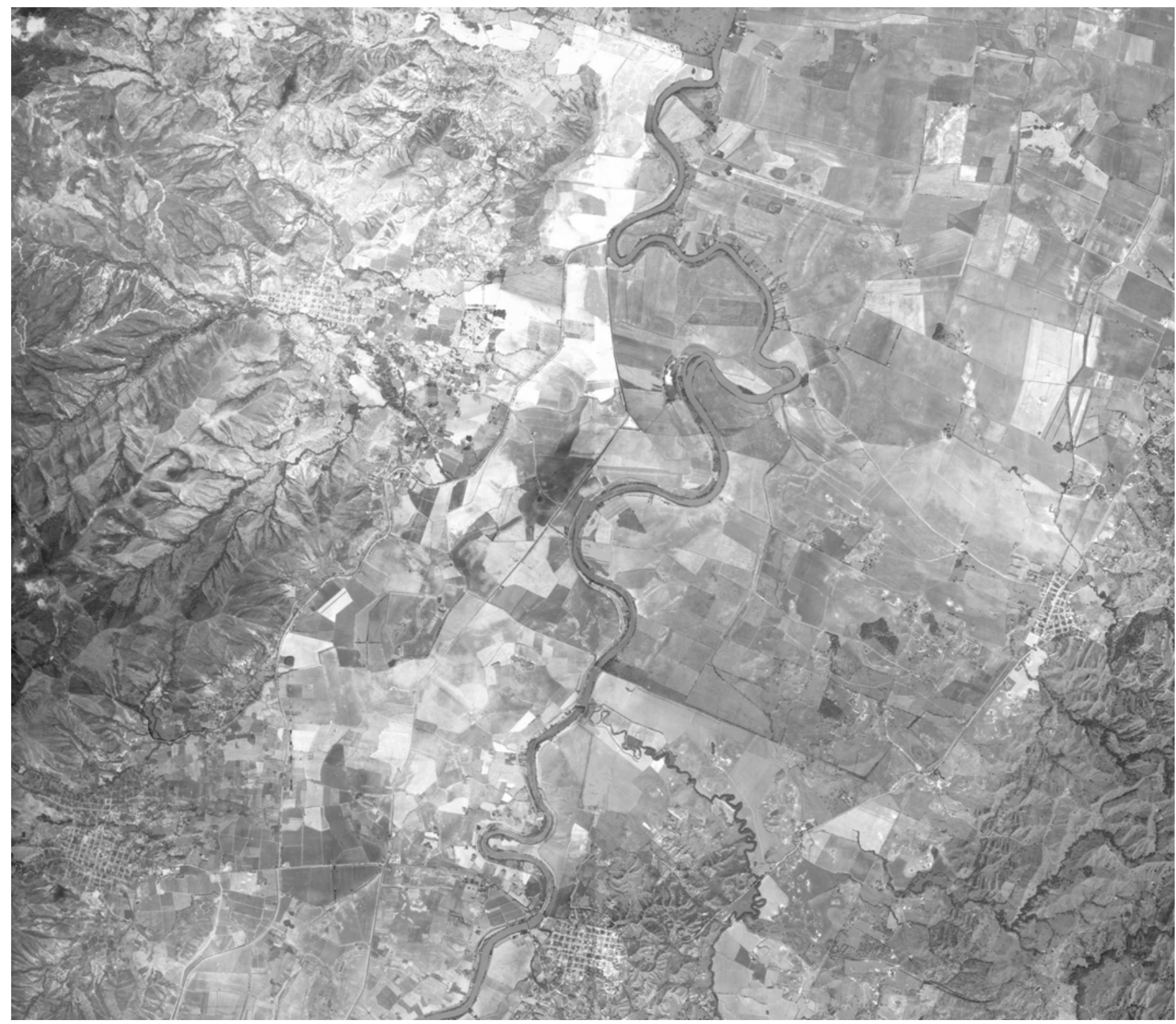

Figura 2. Mosaico del sector de estudio (escala 1:100.000)

colores oscuros, debido a la presencia de buena cantidad de materia orgánica en sus suelos; y por la ausencia de gravilla y fracciones gruesas.

En las proximidades de la localidad de Obando, fue identificado un abanico de edad reciente (ACs23) cubriendo parcialmente al subreciente mencionado anteriormente. Este abanico más joven, tiene su ápice donde la Quebrada de Obando deja la parte montañosa para ingresar al área de piedemonte. Los materiales que componen esta unidad son de granulometría media con presencia, en algunos sectores, de gravilla fina. En las fotografías aéreas presenta tonos claros y en el campo se aprecian colores pardos en la capa arable y en profundidad los horizontes enterrados más oscuros del abanico subreciente. En las proximidades de la localidad de Victoria se reconoció un glacís coluvial (ACs24) de poca extensión, su pendiente oscila entre $7-12 \%$ y está compuesto por materiales predominantemente finos provenientes de los lomeríos (ACs211).
En el piedemonte de la Cordillera Occidental, próximo a la localidad de La Unión, se identificó la parte distal de un abanico subreciente (ACs42) que presenta materiales muy finos, con tonalidades oscuras, y que se encuentra cubierto parcialmente por un abanico de edad subactual (ACs43). Este último, está conformado por materiales de granulometría media y gravilla en algunos horizontes de las parte apical y del cuerpo. En las cercanías del municipio de Toro, se encuentra otro abanico subreciente (ACs42) con iguales características y extensión que el anterior. Entre ambas localidades existe un glacís coluvial (ACs44) de corta extensión constituído por materiales predominantemente finos provenientes de las diabasas de la Cordillera Occidental.

Los piedemontes de ambas cordilleras, constituídos por materiales finos y muy finos (parte distal) muchas veces se confunden con los depósitos aluviales del río Cauca, ya que presentan similar granulometría. Además unos y otros presentaron en distintas épocas, dinámicas diferentes, 
resultando en el área de contacto, sucesivos horizontes provenientes de una u otra unidad.

Tanto en la Cordillera Central como en la Occidental existe una serie de valles estrechos de características coluvioaluviales (ACs32 y BCs12). En algunos sectores estas unidades han ensanchado su valle lo suficiente como para asignarles un carácter aluvial, sin embargo no son muy representativas por la escasa área que ocupan dentro de la zona de estudio.

En el sector más bajo del valle la secuencia de sedimentación del río Cauca y de los tributarios, muestra una alternancia vertical como resultado de la distinta dinámica que presentaron estos agentes a través del Holoceno.

Las geoformas encontradas dentro del plano aluvial corresponden a diques naturales, napas de desborde, basines, brazos deltaícos, cauces antiguos y orillares.

Las áreas de basines, corresponden a las superficies topográficas más deprimidas. En amplias zonas estos basines se encuentran "surcados" por numerosos brazos deltaícos subactuales, que son el resultado de los continuos desbordes del río Cauca hacia estos sitios a través de los vertederos del cauce principal.

Al contarse con fotografías de diferentes años se tuvo la posibilidad de comparar el estado de anegamiento que presentaban algunos sectores del Basín y compararlos con su estado actual. Debido a la regulación del caudal del río aguas arriba de la zona de trabajo y a las obras emprendidas de recuperación de tierras llevado a cabo en dichas superficies, se puede considerar a estas geoformas como no activas, ya que en la actualidad se han controlado los procesos que les dieron origen.

Los cauces antiguos evidencian la continua migración del curso del río dentro del plano de inundación. Estas geoformas pueden observarse claramente en las fotografías aéreas y en el campo, ya que se presentan como zonas alargadas y deprimidas. A ambos lados de estas aparecen sectores un poco más elevados, convexos, que corresponden al dique y napa de estos antiguos cauces.

Los materiales depositados por el río Cauca presentan un gradiente de sedimentación, encontrándose los más gruesos en los cauces antiguos y hacia los basines, los más finos. Los diques y napas se caracterizan por presentar materiales de texturas limosas; con topografías convexas y planas respectivamente; siendo las unidades que tienen mayor diversidad en el uso de la tierra, porque presentan la mejor condición de drenaje. Se localizan a ambos márgenes del río Cauca ocupando franjas estrechas. En cuanto a los orillares observados, presentan formas topográficas cóncavas y convexas, lo que dificulta la mecanización agrícola de tales unidades.

En cuanto a las geoformas degradacionales, éstas se ubican a ambos lados del valle. Se trata de conjuntos montañosos y colinados de distintas edades (Cretáceo-Terciario) y litología, que tienen un rumbo general Noreste-Suroeste, aumentando en altura a medida que se alejan del valle. En estos sectores, las fotografías utilizadas permitieron hacer delimitaciones de las geoformas que allí se presentan, con un alto grado de precisión, siendo de mucha utilidad la visión estereoscópica. Se encontró una alta relación entre los paisajes delimitados y los suelos encontrados, que se manifestaron en la fotografía a través de las distintas variaciones texturales y tonales. La delimitación se basó fundamentalmente tomando en cuenta: el relieve, el patrón de drenaje, el grado y profundidad de disección, y la vegetación.

La resistencia de los distintos materiales y el carácter estructural de una (colinas de la Cordillera Central) y masivo (montañas denudativas de la Cordillera Occidental) de las otras, han dado como resultado distintos grados de erosión y diversas pendientes en estas unidades.

El índice de calidad de Legros (I) calculado dió como resultado un valor de 198,8 y de acuerdo a la escala del mapa final obtenido (1:50.000) y a los rangos que figuran del mencionado índice en la Tabla 2 se concluye que la calidad del mismo es excelente.

Tabla 2. Calidad del mapa de suelos de acuerdo al índice de Legros

\begin{tabular}{rccc}
\hline \multirow{2}{*}{ Escala } & \multicolumn{3}{c}{ Índice (I) } \\
\cline { 2 - 4 } & Insuficiente & Aceptable & Excelente \\
\hline $1: 10.000$ & $<60$ & $60-90$ & $>90$ \\
$1: 25.000$ & $<70$ & $70-120$ & $>120$ \\
$1: 50.000$ & $<90$ & $90-160$ & $>160$ \\
$1: 100.000$ & $<100$ & $100-200$ & $>200$ \\
\hline
\end{tabular}

\section{CONCLUSIONES}

1. La fotografía aérea desempeñó un papel fundamental en el mapeo de los suelos a nivel semidetallado; ya que la fotointerpretación permitió detectar los fenómenos físicos y antrópicos, que en muchos casos se relacionaron íntimamente con los suelos.

2. Se comprobó la utilidad y eficiencia del análisis fisiográfico y su clasificación por el sistema CIAF, al lograrse una delimitación precisa de los suelos en el levantamiento edafológico semidetallado del área bajo estudio.

3. En la zona plana, los elementos que permitieron el reconocimiento de las unidades de paisaje, son: canales de drenaje, uso de la tierra, condición de drenaje y procesos pedogenéticos que en algunas unidades se manifestaron en las fotografías a partir de los cambios de tonalidades. Es importante destacar que en estos sectores, el relieve, tuvo muy poca importancia debido a que los desniveles en el terreno son de baja magnitud.

4. En la zona de mayor relieve, los elementos que permitieron diferenciar las distintas unidades de paisaje fueron: la configuración de la superficie (relieve y pendiente), vegetación natural, rocas, patrón de drenaje, material parental y condición de la erosión.

\section{AGRADECIMIENTOS}

Los autores agradecen por su colaboración a la Unidad de Suelos de la Subdirección de Docencia e Investigación del Instituto Geográfico Agustín Codazzi (IGAC), Bogotá (Colombia) y a la prof. Nelida Rimondi por la traducción del resumen al inglés. 


\section{LITERATURA CITADA}

Barbosa, O.A.; Bertani, L.A.; Fidelia R. Hidalgo Fernandez; Paez Mendoza R.. Análisis fisiográfico y evaluación de tierras para un sector del Valle del Cauca (Colombia). En: Congresso Brasileiro de Engenharia Agrícola, 26, Anais... . Campina Grande 1997. CD Rom

Barbosa, O.A.; Lartigue, C.; Galarza, F.M.; Balmaceda M.R.; Orozco D.M.; Solari F.A. 2000b. Relevamiento detallado de suelos en pequeñas superficies utilizando el análisis fisiográfico. En: Simposio Latinoamericano de Percepción Remota 9 y Reunión Plenaria SELPER, 19. Actas. Puerto Iguazú. CD Rom

Barbosa, O.A.; Lartigue, C.; Galarza, F.M.; Cerda R.A; Rosatto, H. Mapa de suelos detallado empleando el sistema CIAF. En: Congresso Brasileiro de Engenharia Agrícola, 28, 2000. Anais... . Fortaleza. CD Rom

Barbosa, O.A.; Tognelli G.C.; Solari F.A. \& Lartigue C. Uso de la Tierra para un sector del Río Chorrillos (San Luis, Argentina). En: International Congress on Agricultural Engineering, 13, Proceedings. Rabat, Marruecos. v.1, p.457467. 1998.

Bennema, J.; Gelens H.F. Interpretación de Fotografías aéreas. Bogotá, CIAF-IGAC. 176p. 1976.

Botero, P.J. 1984. Guías para el análisis fisiográfico: Bogotá, CIAF. Colombia. 1984, 88p.

Elbersen, G.W.W.; Benavides S.T.; Botero P.J. Metodología para levantamientos edafológicos. Bogotá: IGAC. 1989. 91p.

Forero, M.C.; León J.C. \& Lopez J. Metodología para levantamientos edafológicos. Bogotá: IGAC. 194p. 1988.
García, R. El clima de Colombia. Revista N.3 “Colombia sus gentes y regiones”. Bogotá: IGAC. 1983. 34p

Gómez H. Estudio geológico - geomorfológico del Valle del Cauca. Bogotá, CIAF-IGAC. 1977. 148p

Gonzalez Bernaldez F. Ecología y Paisaje. Madrid, H. Blume Ediciones. 250 p. 1981.

López G.; Olaya T. Clasificación climática de Colombia. Bogotá: Universidad Jorge Tadeo Lozano. 1985. 205p.

Ministerio de Medio Ambiente de España. Secretaria General de Medio Ambiente. Guía para la elaboración de estudios del medio físico. $4^{\text {ta }}$ reimpresión. Madrid, 809p.

Romero, M. Levantamientos de suelos. parte. Bogotá: IGAC. 1992.55p.

Soil Survey Laboratory Staff. Soil survey laboratory methods manual. Lincoln: USDA-SCS, Natlional. Soil Survey Center. 1996. 716p.

Soil Survey Division Staff. Soil survey manual. Washington, D.C.: USDA 1993. 437p. USDA-SCS. Handbook, No. 18.

Soil Survey Staff. Soil taxonomy. A basic system of soil classification for making and interpreting soil surveys. 2, Washington: US Government Printing Office, DC, USA, 869p. 1999. 716p. Agriculture Handboock No. 436.

van Wambeke, A. Calculated soil moisture and temperature regimes of South America. Washington: USDA-SCS. 1981. 716p. SMSS Technical Monograph, 2

Villota, H. Técnicas modernas de mapeo de suelos de ladera. Bogotá: IGAC. 1992. 32p.

Villota, H. Geomorfología aplicada a levantamientos edafológicos y zonificación física de las tierras. Bogotá: IGAC. 1992. 258p.

Villota H. Una nueva aproximación a la clasificación fisiográfica del terreno. Revista CIAF, Bogotá,v.15, p.83-115. 1997. 